\title{
Enquête sur la contraception d'urgence (CU) au niveau des pharmacies
}

\author{
Nafissatou J. Diop \\ Population Council \\ Babacar Mane \\ Ousseynou Faye \\ Cheikh Ibrahima Niang \\ Thiaba Thiaw
}

See next page for additional authors

Follow this and additional works at: https://knowledgecommons.popcouncil.org/departments_sbsr-rh

Part of the Demography, Population, and Ecology Commons, Family, Life Course, and Society Commons, Gender and Sexuality Commons, International Public Health Commons, Medicine and Health Commons, and the Women's Health Commons

How does access to this work benefit you? Let us know!

\section{Recommended Citation}

Diop, Nafissatou J., Babacar Mane, Ousseynou Faye, Cheikh Ibrahima Niang, Thiaba Thiaw, Aissatou Sano Coly, Fatou Mbow, and Youmane Niang Faye. 2009. "Enquête sur la contraception d'urgence (CU) au niveau des pharmacies," Rapport final [executive summary in English; report in French]. Dakar: Population Council. 


\section{Authors}

Nafissatou J. Diop, Babacar Mane, Ousseynou Faye, Cheikh Ibrahima Niang, Thiaba Thiaw, Aissatou Sano Coly, Fatou Mbow, and Youmane Niang Faye 
RéPUblique du SÉnÉGAL

Ministère de la Santé et de la Prévention

Direction de la Santé

Division de la Santé de la Reproduction

\section{Enquête sur la Contraception}

d'Urgence (CU)

au niveau des pharmacies

Septembre 2009

(2 Population Council 



\title{
Enquête sur la Contraception d'Urgence (CU) au niveau des pharmacies
}

\author{
Nafissatou J. DIOP, Ph.D \\ Babacar MANE \\ Dr Ousseynou FAYE \\ Dr. Cheikh NIANG \\ Thiaba THIAW \\ Aissatou SANO COLY \\ Fatou Bintou MBOW \\ Youmané NIANG FAYE
}




\section{TUDY SUMMARY}

\section{CONTEXT}

The Division of Reproductive Health (DRH) with assistance from the Population Council has in recent years conducted an operational research program on Emergency Contraception (EC). The objective of this research has been to provide baseline information for use in developing a strategy for the promotion of EC use in Senegal. Studies have shown that rapid access to an EC method would lead to a significant reduction in the extent of unwanted pregnancies and abortions among women. However, despite the fact that $\mathrm{WHO}$ has recommended the integration of EC into national health programs, access to this method is still very limited for the vast majority of women around the world. In Senegal, studies conducted in 2003 and 2006 revealed that there was little public knowledge about EC and that pharmacies remained the main outlet through which this method was offered.

\section{GENERAL OBJECTIVE}

To propose a strategy aimed at improving access through pharmacies to Emergency Contraceptives.

\section{SPECIFIC OBJECTIVES}

1. Evaluate the availability and the quality of EC services offered through private pharmacies;

2. Evaluate the knowledge and perceptions of the providers and the customers (male and female) about EC;

3. Make Recommendations.

\section{METHODOLOGY}

This study uses a qualitative approach. Qualitative interviews were conducted with provider pharmacies and customers (male/female) at 22 pharmacies in the region of Dakar. The technique of a mystery shopper was also employed to assess the quality of service offered the pharmacy level. A total of 19 interviews were conducted with providers, 76 direct interviews with male and female customers, and 15 interviews with mystery shoppers.

\section{KEY RESULTS}

\section{AVAILABILITY OF EC}

In almost all the pharmacies visited, it is Norlevo ${ }^{\circledR}$ that is available. It is sold freely. The classic conventional Pill is also available and sold by prescription but it is very seldom proposed as a method of EC. The majority of providers are unaware that the conventional Pill could be used as a method for EC.

\section{KNOWLEDGE OF EC AND OF CURRENT PROTOCOLS BY THE PROVIDER PHARMACIES}

Norlevo ${ }^{\oplus}$ is the method most often cited and proposed to customers. Other methods (Pill and IUD) are rarely mentioned. The majority of providers do not use the current information regarding the existing medical protocols on the supply of EC. For example, most do not know that the limit for the effectiveness of the method is up to 120 hours rather than 72 hours. They (the providers) usually refer to the protocol notices when they are offering the EC. This is the result of a lack of training regarding the supply of this method. 


\section{INTERACTION BETWEEN THE PROVIDERS AND CUSTOMERS (MALE/FEMALE)}

The welcome the customers receive remains inadequate and it is clear from statements made that "the sellers do not take the time to speak with the customers, they talk very little and thus don't give really full explanations" in particular as it concerns side effects, protection from STI/AIDS...in addition, the confidentiality rules are not respected with the clients.

\section{COST OF THE METHOD}

The selling price of Norlevo ${ }^{\circledR}$ in pharmacies ranges from 3,200 FCFA and 3,700 FCFA. However, given the exceptional and urgent nature of EC, the cost for the method does not seem to be a major obstacle, "...customers seem to care little about the cost, whether it's high or not, at the moment when their need is satisfied."

\section{PERCEPTIONS OF PROVIDERS ON THE USE OF THE METHOD}

Favorable opinions expressed justify the need for using EC as a method for avoiding unwanted pregnancies especially among young girls. "They help in those instances, those stupid situations of youth to help avoid unwanted pregnancies." (Seller) EC is also perceived as a means for the regulation of births in cases of forgetfulness or error in the framework of regular contraceptive use but among adult married women "it is a right for adult women to use it" (Pharmacist Assistant). Concerning the use of EC by adolescents, the positive arguments put forth maintain "Adolescents are more exposed to early pregnancies and the related dangers, thus they must absolutely be made aware about the use of EC." (Seller).

In contrast, negative opinions about the use of EC are based on beliefs that if it is done in an abusive way, EC use could result in health risks and sexual debauchery... "STI/AIDS are going to continue to wreak their havoc because people will not any longer have the fear of getting pregnant" (Seller). "Adult women should understand that EC should not be taken each time. They should learn to use Family Planning." (Seller). The negative opinions about the use of EC by adolescents also emphasize that "the religion of Islam recommends youth to remain virgins until marriage." (Pharmacist)

\section{PERCEPTIONS OF CUSTOMERS ON THE UTILIZATION OF THE METHOD}

Customers expressing favorable opinions about the use of EC, justify them on the basis that this is an effective method for preventing unwanted pregnancy in emergency situations (forgetfulness/postrape care) especially among young girls, and that it is a right for couples: ..."it's good for couples in committed relations who have made a mistake or have forgotten to calculate their period of fertility." (Laborer) Others argue that the use of EC contributes to empowerment in terms of decision making for women: "It is a good initiative that gives women a voice in deciding for themselves their maternity" (Waitress). Concerning adolescents, those in favor of EC use believe that: "If they do not use it, they run the risk of unwanted pregnancy which could ruin their lives." (Customer, unemployed)

Those expressing a negative opinion link the use of $\mathrm{EC}$ to sexual debauchery and also state that it is in conflict with their religious beliefs: "The youth are delivering themselves up to sexual vagabondage and it is forbidden by Islam." (Businessman)

\section{RECOMMENDATIONS}

- Orient and train pharmacy providers on the new medical protocols in the supply of EC;

- Make available to provider pharmacies other materials related to offering Reproductive Health services;

- Strengthen awareness about EC;

- Improve financial accessibility of EC. 
L'équipe de recherche adresse ses sincères remerciements à tous ceux qui ont contribué à la réalisation de ce travail.

Cette étude a été réalisée grâce au partenariat avec le Ministère de la Santé et de la Prévention ainsi que l'appui technique et financier de Population Council / ECafrique.

Nous saluons ici l'adhésion et la disponibilité du personnel des officines privées qui ont facilité le déroulement de l'enquête et contribué positivement au succès de cette étude.

Soient également remerciés les enquêtrices et enquêteurs ainsi que le personnel d'appui qui ont fait preuve d'un engagement sans faille pour la réussite de cette étude.

Nos remerciements vont également à l'endroit de Madame Fatou Bintou Mbow qui a assuré l'exploitation des questionnaires et l'élaboration du premier draft du rapport.

Que toutes les autres personnes qui ont apporté leur précieuse contribution à la réalisation de ce travail trouvent ici l'expression de notre sincère reconnaissance. 


\section{ABLE DES MATIERES}

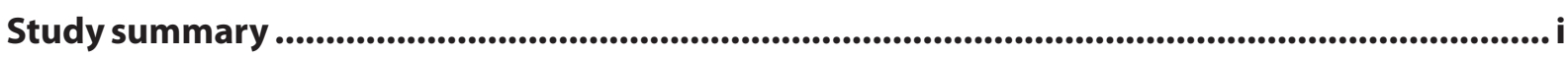

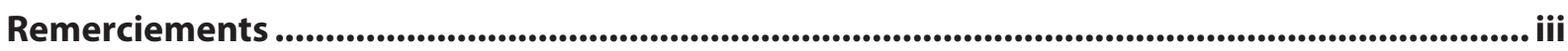

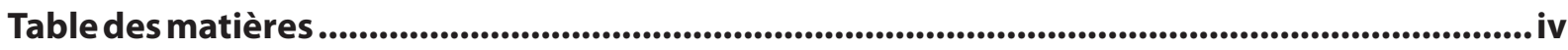

Liste des abréviations/acronymes ............................................................................................................................v

Contexteetjustification ....................................................................................................................................1

Objectifsdel'étude.........................................................................................................................................1 1

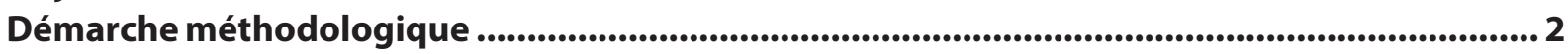

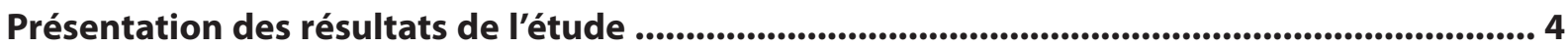

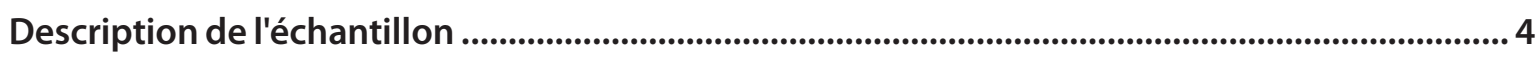

Offre des services de CU dans les officines privées .................................................................................. 4

Disponibilité de la CU ............................................................................................................................................... 4

Connaissance des prestataires des officines sur la CU .............................................................................................. 4

Opinions et perceptions des prestataires des officines sur la $C U$.............................................................................. 5

Qualités des services de CU .......................................................................................................................................

Coût de la CU et son appréciation ............................................................................................................................. 10

Statistiques des ventes de médicaments pour la CU ............................................................................................. 10

Promotion de la CU ................................................................................................................................................. 10

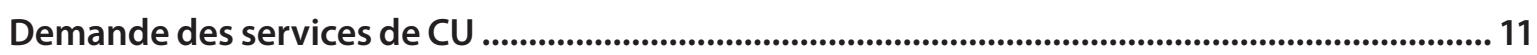

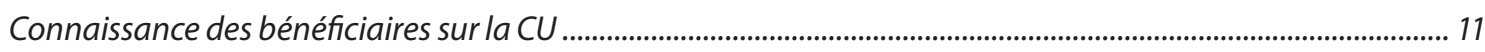

Perceptions/Opinions sur la vulgarisation de la méthode ..................................................................................... 11

Accessibilité de la méthode .......................................................................................................................................... 12

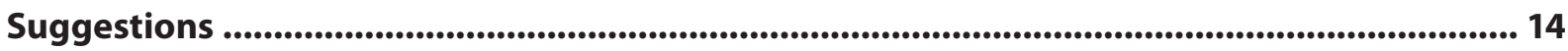

Recommandations ........................................................................................................................... 15

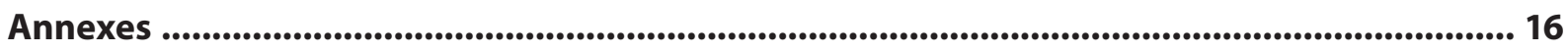

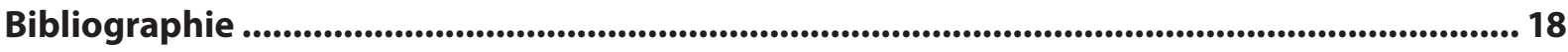

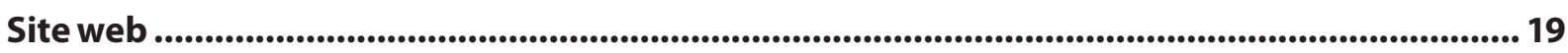




\title{
ISTE DES ABREVIATIONS / ACRONYMES
}

\author{
ADEMAS Agence de Marketing Social \\ ASBEF Association Sénégalaise pour le Bien-être Familial \\ CCA Centre Conseil pour Adolescents \\ CEFOREP Centre de Formation et Recherche en Santé de la Reproduction \\ CU Contraception d'Urgence \\ DSR Division de la Santé de la Reproduction \\ EDS Enquête Démographique de Santé \\ EVF Education à la Vie Familiale \\ GND Grossesse non désirée \\ IEC Information Education Communication \\ IST Infections Sexuellement Transmissibles \\ IVG Interruption Volontaire de Grossesse \\ PCU Pilule de Contraception d'Urgence \\ PF Planification Familiale \\ SR Santé de la Reproduction \\ UNFPA Fonds des Nations Unies pour les activités en matière de Population \\ USAID Agence des Etats-Unis pour le Développement International \\ VIH/SIDA Virus de I'Immuno Déficience Acquise/ Syndrome de I'Immuno Déficience Acquise
}




\section{ONTEXTE ET JUSTIFICATION}

Le Sénégal, connaît un taux élevé de mortalité maternelle, soit 401/100 000 NV (EDS IV, 2005), qui cache de fortes disparités entre les régions. Les besoins non satisfaits en PF sont également élevés : $33 \%$ [EDS III 1997]; 32 \% [EDS IV 2005]

Et la majorité des femmes ayant un besoin non satisfait se retrouvent avec des Grossesses Non Désirées (GND). Au point de vue sanitaire, ces GND les exposent à des conséquences qui sont souvent mortelles ou bien, à des infanticides ou encore à des séquelles (comme les synéchies, l'infertilité, l'infécondité, etc...) dont la majorité peut être invalidante à long ou moyen termes.

Pour parer à cette situation, une des stratégies majeures de la feuille de route multisectorielle de lutte contre la mortalité maternelle et néonatale repose sur la relance de la PF avec l'élargissement de la gamme des méthodes contraceptives. La promotion de la Contraception d'Urgence (CU) constitue une option prioritaire dans le cadre de cette stratégie.

Population Council, en partenariat avec le Ministère de la Santé a mené depuis 2005 différentes actions dans le cadre de la promotion de cette méthode qui était jusque là mal connue des populations et pas assez prise en charge.

Ainsi, plusieurs études (CEFOREP $2003^{1}$; DSR/Population Council $2006{ }^{2}$ ) ont montré que cette méthode contraceptive d'exception demeure encore peu connue du public. Elles ont également fait le point sur la disponibilité de la CU et ont proposé des stratégies pour un meilleur accès de cette méthode et par conséquent une amélioration de son utilisation.

Le Ministère de la Santé a entrepris une série d'actions pour améliorer l'offre de la CU :

- Renforcement des compétences des prestataires et des relais communautaires;

- Partenariat avec I'UNFPA qui a permis de mettre le produit à la disposition des structures de santé ;

- Développement d'outils IEC destinés aux prestataires de santé et à la communauté ;

- Plaidoyer à l'occasion de journées médicales ;

- Développement d'un kit de réponses rapides sur la CU.

En plus d'être disponible dans le secteur public, la CU est largement disponible au niveau des officines privées où cette méthode est en vente libre. Il s'avère d'ailleurs que c'est le principal canal d'obtention de la CU. En effet, au niveau d'une des centrales d'achat (SODIPHARM), il est enregistré entre janvier et juillet 2009 une sortie moyenne mensuelle de 965 boites de Norlevo ${ }^{\oplus}$, nom de commercialisation d'une PCU.

La CU étant une méthode d'urgence et d'exception devant aider les femmes/les couples à aller vers une protection régulière, il apparaît nécessaire de faire le point sur l'offre de services dans les pharmacies.

\section{OBJECTIFS DE L'ETUDE}

\section{Objectif général}

L'objectif principal de cette étude est de proposer une stratégie visant à améliorer l'offre de la CU au niveau des pharmacies.

\section{Objectifs spécifiques}

De manière plus spécifique, il s'agit de :

- Évaluer les connaissances/perceptions/opinions des pharmaciens, vendeurs en pharmacie et des client(e) s sur la CU;

- Évaluer la disponibilité, l'accès et la qualité de l'offre des services de CU dans les pharmacies ;

- Identifier les informations clés fournies aux client(e)s qui demandent la CU au niveau des pharmacies ;

- Identifier des stratégies d'orientation vers les autres services de SR ;

- Formuler des recommandations pratiques pour améliorer l'offre de la CU dans les pharmacies.

1 CEFOREP, Etude exploratoire sur la contraception d'urgence au Sénégal, 2003

2 Population Council,/Equilibres \& Populations/ECafrique, Etude Diagnostique sur la Contraception d'Urgence, 2006. 


\section{EMARCHE METHODOLOGIQUE}

Cette étude a utilisé une approche participative et s'est basée sur des méthodes de collecte d'informations essentiellement qualitatives.

\section{Sites d'étude}

L'enquête a été réalisée dans la région de Dakar. Au total vingt deux (22) pharmacies ${ }^{3}$ choisies au hasard ont été visitées. II s'agit de pharmacies assez bien fréquentées, proche d'un lycée, d'une structure de santé ou d'un marché, situées aussi bien au cœur de la capitale que dans la banlieue Dakaroise.

\section{Population cible de l'étude}

L'étude s'est particulièrement orientée vers trois cibles:

- Le personnel des officines privées (pharmaciens, vendeurs en pharmacie et caissiers) ;

- Les client(e)s qui fréquentent les pharmacies pour l'achat de n'importe quel médicament ;

- Les client(e)s "mystère". Ce sont des client(e)s issu(e)s des groupements féminins, des ASC, des universités... qui ont été formés pour assurer le rôle de vrais clients et simuler un besoin de CU.

\section{Echantillonnage}

Dans l'ensemble, 110 personnes ont été interrogées. En ce qui concerne les client(e)s "mystère", ils ont chacun visité 3 pharmacies différentes, d'où un total de 140 entretiens individuels qui ont été menés dans le cadre de cette étude.

\begin{tabular}{|c|c|c|c|}
\hline \multirow[b]{2}{*}{ Cible } & \multicolumn{2}{|c|}{ Personnes touchées } & \multirow[t]{2}{*}{ Total } \\
\hline & Hommes & Femmes & \\
\hline Pharmaciens & 5 & 4 & 9 \\
\hline Assistants en pharmacie & 0 & 1 & 1 \\
\hline Vendeurs & 8 & 0 & 8 \\
\hline Caissièr(e)s & 0 & 1 & 1 \\
\hline Client(e)s "mystère" & 9 & 6 & 15 \\
\hline Client(e)s & 42 & 34 & 76 \\
\hline Total & & & 110 \\
\hline
\end{tabular}

\section{Outils de collecte}

Trois (3) outils ont été développés pour la collecte des informations:

- Un guide d'entretien à l'intention des pharmaciens et vendeurs en pharmacies.

Cet instrument a été administré à toute personne qui officie dans la pharmacie quelque soit son statut. Les questions ont porté sur leurs connaissances de la CU, l'offre des services, I'utilisation de la $\mathrm{CU}$, les statistiques de vente et sur leurs suggestions.

- Un guide d'entretien à l'intention des client(e)s "mystère".

Cet outil, adressé à la sortie de la pharmacie a surtout permis de recueillir toutes les informations relatives à l'offre de services de CU, à la disponibilité des produits de CU mais aussi les conseils et orientations formulés par les personnes officiant au niveau des pharmacies.

- Un guide d'entretien pour les client(e)s qui fréquentent les officines de pharmacie.

Ce guide a été adressé aux client(e)s qui ont visité la pharmacie durant l'enquête. II a permis de collecter des informations sur leurs connaissances/perceptions de la CU, I'utilisation de la CU, la qualité et la disponibilité des services, ainsi que leurs suggestions. 


\section{Organisation de la collecte des données}

Les objectifs de l'étude ont été partagés avec les autorités du Ministère de la Santé et plus particulièrement avec la Division de la Santé de la Reproduction. De même, les outils de collecte ont été développés avec les services de ce Ministère.

La collecte des données s'est déroulée du 13 au 20 juillet 2009 et s'est effectuée en 2 phases :

1. Le passage des client(e)s "mystère" du 13 au 15 juillet 2009

2. L'enquête proprement dite du 17 au 20 juillet 2009.

L'équipe d'enquêteurs était constituée de 5 enquêteurs professionnels (dont 3 femmes et 2 hommes) pour les pharmaciens, vendeurs en pharmacie et caissiers, ainsi que pour les client(e)s qui ont fréquenté les officines durant la période de l'enquête.

Les client(e)s "mystère" entièrement constitués d'enquêteurs professionnels étaient au nombre de 15 dont 6 femmes et 9 hommes. 10 d'entre eux ont entre 20 et 27 ans, et 5 ont entre 32 et 49 ans. Ils sont composés de Wolof (4), de Diola (4), de Hal pulaar (3) et de Sérère (2) pour l'essentiel. Ils sont pour la majorité de célibataires (10), le reste étant marié.

Chaque client "mystère" a fait un total de trois visites dans trois pharmacies différentes, d'où un total de 45 sorties à l'occasion desquelles la plupart d'entre eux se sont retrouvés face à un vendeur (25), ou face à un pharmacien (15). Le reste a été reçu par des caissiers ou autres.

La collecte des données a été supervisée par une équipe composée de deux personnes : un représentant du Ministère de la Santé et la coordinatrice du projet CU de Population Council.

\section{Considérations éthiques}

La participation des différents groupes aux enquêtes était strictement volontaire. Pour s'assurer que l'étude s'est faite selon les normes de l'éthique, un formulaire de consentement éclairé a été intégré aux questionnaires pour obtenir le consentement de chaque personne interviewée. Toutes les données collectées ont été traitées de manière confidentielle. Des mesures ont été prises pour assurer le respect de la dignité et la liberté de chaque individu invité à participer. Les noms des participants n'ont pas été marqués sur les questionnaires pour garantir l'anonymat

Les noms des pharmacies visitées également n'ont pas été mentionnés.

\section{Méthode d'analyse}

L'Etude s'est basée sur des méthodes essentiellement qualitatives. Etant donné que le but de cette enquête est de proposer des stratégies pour améliorer l'offre de la CU aux client(e)s au niveau des pharmacies, l'approche a porté essentiellement sur une évaluation de l'état des informations sur la base d'une analyse de contenu. L'ensemble des données issues des différents outils de collecte a fait l'objet d'une analyse par thème approfondie. 


\section{RESENTATION DES RESULTATS DE L'ETUDE}

\section{DESCRIPTION DE L'ECHANTILLON}

\section{Les prestataires des officines privées}

Les 19 personnes interviewées officiant dans les officines de pharmacie sont dans leur majorité constituée d'hommes (13). Les 6 femmes interviewées sont légèrement plus âgées que les hommes, en effet leur âge moyen est de 43 ans alors qu'il n'est que de 40 ans pour les hommes.

L'échantillon est composé de wolof (8), de sérère (3), de Hal pulaar (2), et de Diola (4) pour l'essentiel. Ils sont pour la plupart mariés (73,7\%), neuf (9) d'entre eux sont titulaires d'un doctorat en pharmacie. Les vendeurs et caissiers représentent $47 \%$ de l'échantillon.

\section{Les client(e)s}

Dans le cadre de cette étude, au total 76 client(e)s ont été interviewé(e)s dont 42 hommes et 34 femmes. Les données montrent que les client(e)s rencontré(e)s durant l'enquête sont relativement jeunes avec un âge moyen de 33 ans. Il faut cependant mentionner qu'une grande disparité est notée aussi bien chez les hommes que chez les femmes avec des âges qui fluctuent entre 14 et 59 ans chez les hommes et de 18 à 64 ans chez les femmes. La répartition ethnique indique que l'échantillon est représenté de wolof (35) et de Hal pulaar (15), de sérère (13), de Diola (5) et de Mandingue (4) pour l'essentiel. La plupart d'entre eux sont mariés (46), les célibataires représentent 35,5\% de l'échantillon.

\section{OFFRE DE SERVICES DE CU DANS LES OFFICINES PRIVEES}

\section{DISPONIBILITE DE LA CU}

Dans la quasi-totalité des officines visitées, c'est la CU sous forme de Pilule de Contraception d'Urgence (PCU) qui est disponible, et particulièrement le Norlevo ${ }^{\oplus}$. Cette disponibilité était effective durant le dernier trimestre précédant la collecte de données, hormis au niveau de deux d'entres elles.

La méthode Yuzpe ${ }^{4}$ est très peu proposée alors que les pilules classiques sont disponibles au niveau des pharmacies. Seules 5 pharmacies déclarent proposer la méthode Yuzpe sous forme de CU, mais seulement lorsque celle-ci fait l'objet d'une prescription médicale.

Ainsi une ordonnance est exigée pour disposer de cette dernière méthode, contrairement au Norlevo ${ }^{\oplus}$ qui est en vente libre car inscrite au tableau des médicaments simples.

\section{CONNAISSANCE DE LA CU PAR LES PRESTATAIRES DES OFFICINES PRIVÉES}

Chez les prestataires des officines privées, la CU est bien connue dans la mesure où ils ont tous déclaré connaître l'existence de ce produit. Mais elle est surtout connue sous l'appellation "Pilule du lendemain".

Ce qu'il faut noter, c'est que cette connaissance se limite principalement à la PCU, plus précisément le Norlevo ${ }^{\oplus}$, qui est, comme il a été indiqué plus haut, pratiquement la seule méthode de CU proposée dans la plupart des officines du secteur privé.

En ce qui concerne la posologie relative à la $\mathrm{CU}$, tous les prestataires des officines déclarent la connaître. En fait, ils assurent ne pas disposer de protocoles et se réfèrent pour la majorité, sinon tous, à la notice du produit quand ils offrent la CU.

"Nous ne disposons pas spécifiquement de protocoles, mais on se réfère à la notice" 
La plupart des prestataires n'ont pas d'informations actualisées sur les protocoles en vigueur concernant l'offre de CU. Par exemple, la grande majorité ne sait pas que le délai d'efficacité de la méthode pouvait aller jusqu'à $120 \mathrm{~h}$ au lieu de $72 \mathrm{~h}$ (selon la Déclaration du Consortium International pour la Contraception d'Urgence de 2003). Cette situation s'explique par le manque de formation sur l'offre de cette méthode

\section{OPINIONS ET ATTITUDES DES PRESTATAIRES DES OFFICINES SUR LA CU}

La majorité des prestataires des officines privées (11/19) pense que la CU est une bonne méthode et qu'elle devrait être vulgarisée ne seraitce que pour informer la communauté de son existence. De plus, cela permettra de mieux spécifier l'intérêt de cette méthode d'exception par rapport aux autres méthodes de contraception. Elle permettra également de la distinguer de l'IVG car certains la considèrent comme telle.

Cette adhésion s'explique de plusieurs manières, comme ils ressort des commentaires des personnes interviewées.

C'est une méthode efficace qui permet d'éviter les grossesses non désirées surtout chez les jeunes filles.

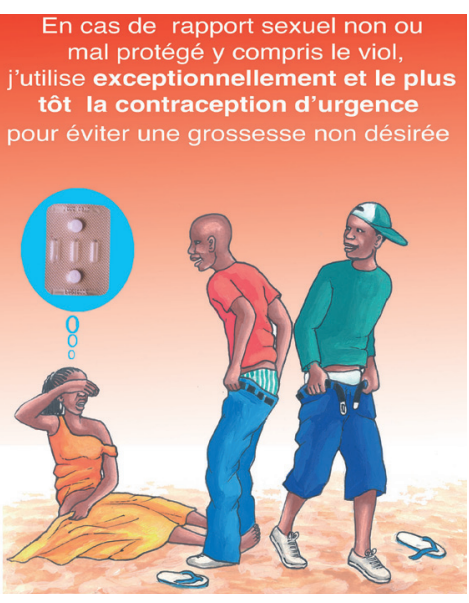

"Elle arrange les situations, les bêtises des jeunes pour éviter les grossesses non désirées"

(Vendeur en pharmacie)

C'est un moyen de réguler les naissances en cas d'oubli, d'échec ou d'erreur dans le cadre de la contraception régulière.

C'est aussi un moyen de pallier à la rupture de préservatif qui est quelque fois observée au niveau de certaines pharmacies.

"Bonne utilité car étant une autre forme de contraception normale car il y a souvent rupture de préservatif"

(Vendeur en pharmacie)

C'est pourtant ce rôle de substitution que certains déplorent du fait des conséquences que cela pourrait engendrer par rapport à l'adoption de comportements à risque lié à l'absence de protection contre les IST/SIDA.

"Les IST/SIDA vont continuer à faire des ravages car les gens n'auront plus peur de tomber enceinte"

(Vendeur en pharmacie)

D'autres justifient leur désapprobation par rapport à cette méthode du fait de l'abus dont son utilisation peut faire l'objet et du fait également des effets pervers qu'elle pourrait susciter en matière de sexualité.

"Je suis contre la CU car elle se vend comme de petits bonbons et elle favorise la débauche"

(Pharmacien)

\section{Opinions des prestataires sur I'utilisation de la CU par les bénéficiaires}

Les prestataires dans leur majorité semblent plus favorables à l'utilisation de la CU chez les femmes adultes et mariées plutôt que chez les adolescentes. En effet, l'utilisation de la CU est mieux tolérée chez les femmes adultes mariées, parce que non seulement elles en ont le droit, mais surtout parce qu'il s'agit de planifier les naissances.

"C'est un droit pour les femmes adultes de l'utiliser" 
Pourtant ceux qui sont contre l'utilisation de cette méthode par les femmes adultes se basent sur un argument proche en soutenant que cela pourrait créer la confusion chez certaines.

"Les femmes adultes doivent comprendre que la CU ne doit pas être prise à chaque fois, elles doivent utiliser le planning familial"

(Vendeur en pharmacie)

Par contre la grande majorité des prestataires sont opposées à l'utilisation de la CU par les adolescents. En effet, près des $3 / 4$ des prestataires des officines s'opposent formellement à l'utilisation de la CU chez les adolescent(e)s, non seulement parce que cela peut les inciter à la débauche, mais aussi parce que cela va à l'encontre de certaines valeurs traditionnelles et préceptes religieux.

"La religion musulmane recommande aux adolescentes de rester vierges jusqu'au mariage"

(Pharmacien)

"Il faut plutôt éduquer les jeunes sur l'abstinence. Ils ne doivent pas en avoir besoin car cela peut favoriser la délinquance sexuelle"

(Pharmacien)

Il faut tout de même souligner que certains prestataires, même s'ils ne sont pas assez nombreux, pensent que les adolescent(e)s, souvent victimes de grossesses précoces constituent la cible privilégiée de la CU. Toute fois cette utilisation de la CU par les adolescents doit aller de paire avec la mise en place de garde-fous.

"Les adolescents sont les plus exposés aux grossesses précoces et dangereuses, donc ils doivent être impérativement sensibilisés à l'utilisation de cette CU"

(Vendeur en pharmacie)

"Les adolescentes peuvent prendre la CU, mais doivent être suivies par des centres de planning familial pour une meilleure sensibilisation"

(Vendeur en pharmacie)

\section{Attitudes des prestataires des officines face aux demandeurs de la CU}

L'attitude des prestataires sur l'utilisation de la CU se reflète sur leurs réactions face aux demandeurs de la CU. Si l'on se base sur les propos des prestataires des officines, ils disent avoir, pour la grande majorité, des réactions plutôt positives lorsqu'ils reçoivent des client(e)s qui demandent la CU dans la mesure où aucun refus n'a été noté du moins en ce qui concerne les femmes adultes. Les services à l'endroit de ces dernières sont offerts sans grande difficulté. Mieux, la vente du produit est souvent accompagnée de conseils et d'explications selon eux. Cette attitude à l'endroit des femmes adultes est même affirmée chez les rares prestataires qui affichent quelques réticences compte tenu de certaines de leurs convictions.

"J'accueille et j'informe sur le BERCER ${ }^{5}$, les avantages et inconvénients de la méthode"

(Pharmacien)

"Je suis choqué car j'ai toujours préféré la vie et donc contre la contraception et la CU, mais néanmoins, je leur parle et leur vend le produit"

(Vendeur en pharmacie)

Par contre c'est sont les adolescent(e)s qui font parfois l'objet de stigmatisation, voire de rejet même si la plupart des prestataires des officines déclarent leur offrir une prise en charge adéquate.

En effet, pour s'en "débarrasser" semble-t-il, certains usent souvent de subterfuges tels que la référence vers les centres de PF ou Centre Conseils Ados sous le prétexte de leur faire bénéficier disent-ils de plus amples informations.

"Pour les adolescents, je leur demande de se rendre au centre ado ou au centre de PF" 
"Nous les orientons vers les centres ASBEF ou Centre Ado pour qu'ils soient mieux informés sur la $C U^{\prime \prime}$

(Vendeur en pharmacie)

D'autres prestataires s'arrogent tout simplement le droit de réglementer l'offre de la CU en exigeant un âge minimum, voire, l'approbation du mari pour deux (2) d'entre eux, ou conditionnent l'offre du produit à la délivrance d'une ordonnance.

"Je demande la date du dernier rapport, la situation matrimoniale et l'âge du client"

(Vendeur en pharmacie)

"Pour le Norlevo ${ }^{\oplus}$, nous donnons seulement aux adultes"

(Pharmacien)

"J'exige un âge minimum parce qu'on ne peut pas permettre aux gens de faire n'importe quoi"

(Pharmacien)

Les déclarations des client(e)s "mystères" montrent en effet qu'avec les prestataires des officines privées, la notion d'urgence en ce qui concerne la contraception en question est le plus souvent dénuée de tout son sens. Alors que la CU est une méthode d'urgence qui doit être prise le plus tôt possible pour garantir une efficacité optimale, on s'aperçoit que des retards sont quelques fois occasionnés par l'exigence d'une ordonnance ou par l'orientation du client qui se fait parfois au début, dès l'exposé du problème par le/la client(e).

Certains prestataires lient la pratique de cette méthode à la débauche sexuelle et au fait qu'elle est contraire aux préceptes de la religion).

"Après explication de mon problème, il m'a demandé d'aller voir la sage femme"

(Cliente "mystère", 22 ans)

"Je suis allée vers une autre pharmacie parce que la vendeuse que j'ai trouvée m'a référé à l'ASBEF»

(Cliente "mystère", 21 ans)

"Le pharmacien n'a pas voulu fournir d'informations. Il m'a demandé une ordonnance prescrite par un gynécologue"

(Client "mystère", 22 ans)

\section{QUALITÉ DES SERVICES DE CU}

Cette qualité des services est ici appréhendée à travers des éléments proposés par le "Modèle Bruce/Jain" 6 :

\section{Le choix de la méthode}

II constitue l'élément fondamental de la prestation de services de qualité selon ce modèle. Et le choix du client devient plus varié lorsque les officines peuvent offrir plusieurs méthodes de CU. Ce qui n'est pas Contraception d'Urgence:CU le cas puisque le produit offert résulte d'une promotion exclusive du Norlevo ${ }^{\circledR}$ qui est pratiquement la seule méthode de CU disponible au niveau des pharmacies visitées.

"Le vendeur m'a dit qu'il n'y a aucune autre méthode de CU que le Norlevo ${ }^{\oplus "}$

(client "mystère")

"On remarque que dans la plupart des pharmacies il n'y a que le Norlevo"

(client "mystère")

Concernant le système d'approvisionnement, il faut noter que le Norlevo est commandé par l'intermédiaire des grossistes.

6 Le "Modèle de Bruce/jain" qui s'articule autour de 6 éléments a été publié par Judith Bruce en 1990 de paire avec des outils de mesure et d'évaluation mis au point par Anrudh Jain. 


\section{Les informations communiquées aux client(e)s}

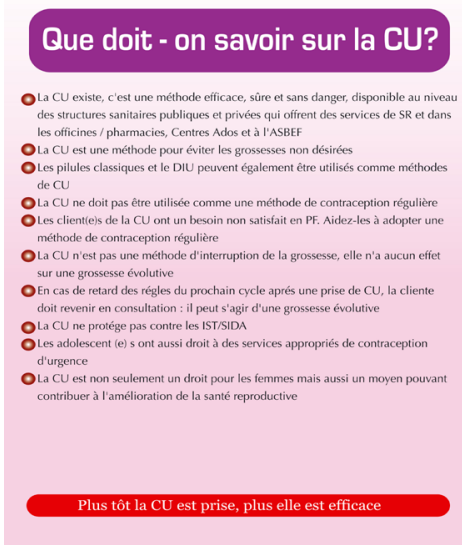

Les prestataires doivent fournir des renseignements complets, clairs et précis pour aider les client(e)s à prendre les meilleures décisions. Mais il est apparu que la vente du produit est rarement accompagnée de conseils ou d'informations comme le prétendent certains prestataires des officines privées.

En effet, selon les client(e)s "mystère", ceux qui fournissent des renseignements exhaustifs sur la CU ne sont pas nombreux car seuls 14/45 ont été informés sur le fait que la CU n'est pas une interruption volontaire de grossesse. 6 client(e)s "mystère" seulement déclarent avoir été informé(e)s du fait que la CU ne protège pas des IST/SIDA, et seulement 10 d'entre eux ont eu des explications sur les effets secondaires de la méthode.

"Les vendeurs ne prennent pas le temps de parler avec les client(e)s. Ils parlent peu, et donc pas d'explications complètes"

(client(e) "mystère")

De même, comme le révèle certain(e)s client(e)s "mystère" (14/45), les prestataires des officines privées informent rarement sur les autres méthodes contraceptives et sur les sources d'approvisionnement de ces dernières.

"Le vendeur ne m'a donné aucune information sur les autres méthodes contraceptives"

(cliente "mystère")

D'autres informations toutes aussi importantes telles que l'existence d'autres méthodes de CU sont rarement livrées. En effet seuls 6 client(e)s "mystère" déclarent en avoir reçu.

Tableau 2 : Types d'informations reçues par les client(e)s "mystère" sur la CU

\section{Informations reçues}

La CU n'est pas une IVG

La CU ne protège pas contre les IST/SIDA

Informations sur les effets secondaires

Informations sur les autres méthodes de CU

Informations sur les autres méthodes contraceptives

Nombre de client(e)s "mystère" N=45

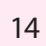

6

6

14

\section{La connaissance des procédures de l'offre de CU}

Malgré une bonne connaissance du produit, près de la moitié des prestataires des officines privées (9/19) déclarent pourtant n'avoir reçu aucune formation, information ou orientation sur la CU. Pour l'autre moitié qui soutient le contraire, il ne s'agit pas en fait de formation "formelle", hormis pour ceux qui ont reçu des orientations dans le cadre de leurs études universitaires. Il s'agit plutôt d'informations reçues par le biais de délégués médicaux, de collègues de travail ou à travers des revues de santé.

Et si l'on se réfère aux propos des client(e)s "mystère", on se rend compte que le personnel des pharmacies ne dispose pas d'informations récentes sur la CU. Ces prestataires se fient naturellement à la notice pour conseiller les client(e)s en ce qui concerne le Norlevo ${ }^{\oplus}$. Ce qui explique l'insistance sur le délai d'utilisation de $72 \mathrm{~h}$.

"Le pharmacien m'a dit que c'était fichu, au-delà de 72h, la CU ne peut rien résoudre, il m'a conseillé d'aller voir un gynécologue»

(client "mystère")

Même si beaucoup d'entre eux affirment que le Norlevo ${ }^{\circledR}$ est à prendre en dose unique dans les 72 heures qui suivent le rapport sexuel douteux, la confusion est encore réelle chez certains en ce qui concerne les délais d'utilisation. 
Ainsi le délai de 5 jours ( 120 heures) pour la prise de la PCU après un rapport sexuel non protégé, n'est encore pas d'actualité chez la plupart des pharmaciens. Ce délai a pourtant été recommandé par le Consortium International pour la CU dans sa déclaration politique de juillet $2003^{7}$. Cette disposition faisant suite aux résultats d'une étude réalisée par l'OMS8.

\section{L'interaction client(e)s-prestataires}

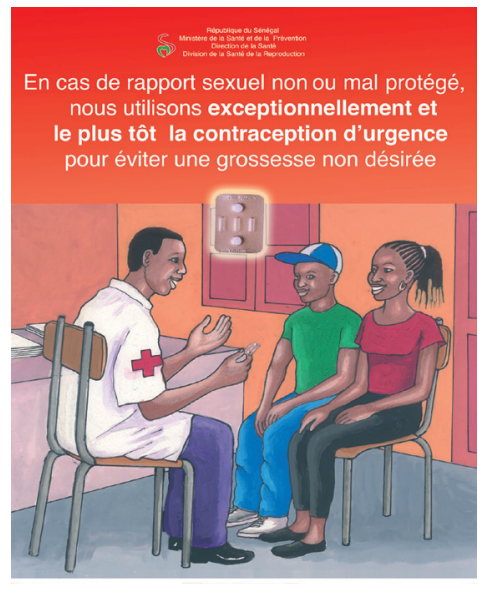

La qualité des services dépend beaucoup de cette interaction. Cependant quelques client(e)s "mystère" se plaignent de n'avoir pas été bien accueilli(e)s, ni avoir été traité(e)s avec respect par certains prestataires des officines privées.

"Le vendeur ne m'a pas accordé d'importance, il me considère comme n'importe quel client"

(client "mystère")

"L'accueil n'était pas aussi intéressant, la vendeuse avait l'air de quelqu'un qui est pressée"

(client "mystère")

"Les vendeurs ne prennent pas le temps de parler correctement avec les clients"

(cliente "mystère")

Certains vont même jusqu'à mettre les client(e)s mal à l'aise en les soumettant à un interrogatoire relatif entre autre à l'âge et à la situation matrimoniale.

"Je suis très choquée et je leur pose énormément de questions et très gênantes parfois"

(Pharmacienne assistante)

\section{Les mécanismes assurant la continuité/Orientation vers les autres services}

La CU étant une méthode ponctuelle, les prestataires doivent s'assurer, après l'offre de ce produit que les client(e)s bénéficient des services de SR dont ils auront besoin ultérieurement. Ce qui suppose une orientation vers les services de PF pour l'adoption d'une méthode de contraception régulière. Cependant l'orientation ou la référence vers les structures de santé et centres de PF n'est pas toujours systématique chez les prestataires des officines. En effet, seule une (1) cliente "mystère" sur les 45 déclare avoir été référée vers une structure de PF après la vente du produit.

"Après, le vendeur m'a suggéré d'aller dans les structures sanitaires pour prendre une méthode de PF car la CU n'est pas à prendre de façon continuelle"

(cliente "mystère")

\section{L'adaptation et l'acceptabilité des services}

L'organisation du service doit répondre aux besoins du client. II faut entre autres que les services soient offerts en toute confidentialité. Les informations recueillies montrent que les prestataires des officines privées se soucient peu des aspects de confidentialité. Seuls 18 client(e)s "mystère" sur les 45 affirment avoir été mis dans un endroit où ils pouvaient s'exprimer en toute confidentialité. Au niveau des officines visitées, on note l'absence d'un espace pour le counseling, ce qui limite la confidentialité des entretiens entre clients et prestataires.

"Il y a un manque de confidentialité des vendeurs»

7 Consortium international pour la contraception d'urgence. Mise à jour sur le régime. Dosage et posologie. Déclaration de politique (juillet 2003). 8 Von Hertzen H, Piaggio G, Ding J, et al. "Low dose mifepristone and two regimens of levonorgestrel for emergency contraception: a WHO multicentre randomized trial." Lancet 2002. 


\section{LE COÛT DE LA CU ET SON APPRÉCIATION}

En ce qui concerne les pharmacies visitées, le coût du Norlevo ${ }^{\circledR}$ varie entre 3200 et 3700FCFA.

Si certains prestataires pensent que pour les client(e)s, le produit est cher, d'autres (9) le trouvent abordable. En fait, les client(e)s se préoccupent peu de la cherté ou non du produit du moment que leur besoin soit satisfait.

"Les client(e)s ne se plaignent pas de la cherté car ils sont pressés de l'avoir"

(Pharmacien)

"Ils ne se plaignent pas du prix, même si on leur taxait 5000FCFA, ils vont l'acheter"

(vendeur en pharmacie)

"Les client(e)s n'hésitent pas à acheter car le plus souvent ils arrivent dans une situation de détresse et de peur de la grossesse"

(Vendeur en pharmacie)

\section{STATISTIQUES DES VENTES DE CU}

Les informations recueillies montrent que les statistiques relatives à l'utilisation de la CU sont rarement disponibles dans les officines. Les données ont été obtenues au niveau de 11 d'entres elles et parmi celles-ci, pour 3 pharmacies, les chiffres donnés ont été avancés de manière approximative ${ }^{9}$.

De manière globale, les données concernent généralement la PCU. Elles sont très disparates et témoignent de sorties variables entre 2 et 264 boites pour le Norlevo ${ }^{\circledR}$ en ce qui concerne les 6 premiers mois de l'année 2009. Pour l'année 2008, les sorties varient entre 15 et 528 boites d'une pharmacie à l'autre.

Pour les autres officines, l'absence des données statistiques est tout simplement due à un manque de traçabilité.

Pour rappel, SODIPHARM a enregistré entre janvier et juillet 2009 une sortie moyenne mensuelle de 965 boites de Norlevo ${ }^{\oplus}$. Et le stock disponible au 31 Juillet 2009 était de 974 boites. Néanmoins tel que l'a montré l'étude diagnostique de Population Council de 2006, la CU reste encore une méthode sous utilisée si I'on se fie aux données recueillies. En effet, sur les 34 clientes interrogées, seules 4 d'entre elles, dont 2 mariées et 2 célibataires, avouent avoir déjà fait recours à la CU sous forme de Norlevo ${ }^{\oplus}$.

Par ailleurs, selon les prestataires des officines privées, la clientèle demandant la CU au niveau des officines est assez variée car il s'agit :

- de jeunes filles surtout, élèves et étudiantes, célibataires et âgées entre 15 et 25 ans ;

- de femmes mariées, le plus fréquemment instruites ;

- de jeunes hommes ayant entre 25 et 35 ans, quelques fois.

\section{PROMOTION DE LA CU}

On note une quasi absence d'activités de promotion de la CU au niveau des officines visitées...En dehors de l'un d'entre eux, aucun prestataire au niveau des pharmacies, ne fait la promotion de la CU. Plusieurs raisons expliquent cette position. Le manque de temps est le facteur dont ils font souvent allusion. Par ailleurs, d'autres facteurs sont évoqués :

Plusieurs d'entre eux pensent que la promotion ne relève pas de leur ressort

"C'est une pharmacie, ce n'est pas son rôle"

(Pharmacienne)

"Nous donnons seulement des conseils aux utilisateurs, on suppose que le médecin a déjà fait le travail de sensibilisation" 
Certains sont contre l'utilisation de ce produit surtout chez les jeunes

"Seuls les jeunes la demandent et je ne suis pas pour les relations extra conjugales"

(Pharmacien)

"Parce que je suis contre, je suis pour l'abstinence chez les jeunes filles"

(Pharmacien)

D'autres encore estiment que la CU est assez connue.

"Le produit fait sa promotion tout seul, les gens le connaissent"

(Vendeur en pharmacie)

\section{DEMANDE DES SERVICES DE LA CU}

\section{CONNAISSANCE DU PRODUIT PAR LES BÉNÉFICIAIRES}

Comme l'ont montré plusieurs études (CEFOREP 2003; DSR/Population Council 2006), l'enquête révèle que la CU demeure jusqu'à présent une méthode peu connue de la population en général. En effet sur les 76 client(e)s interrogé(e)s, seuls 19 déclarent connaître la CU, ou du moins qu'ils en ont entendu parler. Les sources d'informations pour cette cible sont essentiellement constituées de l'entourage, notamment des amis.

En ce qui concerne les lieux d'approvisionnement de la CU, en dehors des officines du secteur privé, la plupart des client(e)s enquêté(e)s pensent trouver la CU non seulement au niveau des hôpitaux, mais également à «Kër Serigne-bi" ${ }^{10}$. D'autres lieux tels que ASBEF, postes de santé, centres de PF, ainsi que ADEMAS ont également été cités.

\section{PERCEPTIONS/OPINIONS DE LA CU PAR LES BÉNÉFICIAIRES}

Une certaine frange de la population des bénéficiaires (22) condamnent la CU parce qu'ils la considèrent comme un avortement volontaire, sinon comme une méthode anti-islamique. Toutefois, il convient de noter que la majorité des client(e)s (54/76) pense que la CU est une bonne méthode du moment qu'elle permet efficacement d'éviter les grossesses non désirées dans des situations d'urgence (exemple oubli) ou en cas de viol surtout chez les jeunes filles. Ils mettent aussi l'accent sur la possibilité qu'elle offre aux femmes d'exercer leur pouvoir de décision en ce qui concerne leur maternité, et de façon globale en matière de sexualité.

"C'est une belle initiative que de donner l'opportunité aux femmes de décider d'elles-mêmes de leur maternité"

(Cliente, restauratrice)

"Je pense que c'est bien pour les couples fidèles qui ont commis une erreur ou oublié de calculer leur période de fécondité"

(Client, maçon)

Pour ceux-là, la vulgarisation est donc nécessaire pour satisfaire, comme l'affirme les prestataires, à des besoins d'informations de la population qui ignore le plus souvent l'existence de ce produit, mais aussi pour apporter une solution face au phénomène de la maternité en dehors du mariage qui est banni de la société sénégalaise. La vulgarisation devient également une exigence pour permettre aux gens, surtout aux femmes de s'épanouir davantage sur le plan sexuel.

Toutefois, certains estiment qu'il est nécessaire, dans le cadre de cette vulgarisation, de poser des limites par rapports aux intervenants.

"La promotion doit être laissée à la charge des pharmaciens et professionnels de santé, mais non aux médias et autres canaux de communication"

(Cliente, vendeuse de légumes)

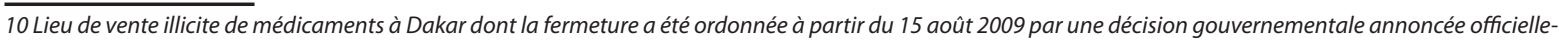
ment par le Premier ministre. 
Par contre, ceux qui ont une mauvaise opinion de la CU justifient leur position de plusieurs manières : selon eux, une large diffusion de cette méthode serait source d'abus, notamment chez les jeunes, mais encouragerait surtout le libertinage sexuel (au détriment de l'abstinence), et parfois l'adultère dans les couples, pratiques qui sont d'autant plus interdites/réprimées par la religion.

"Cela risque d'augmenter l'infidélité dans les couples et freiner l'abstinence"

(Client, chauffeur de taxi)

"Les jeunes vont se livrer au vagabondage sexuel et c'est interdit par I'Islam"

(Client, commerçante)

Comme pour les prestataires des officines, nombreux sont les bénéficiaires qui ne voient pas d'inconvénients à l'utilisation de la CU chez les femmes adultes et mariées, surtout s'il s'agit d'éviter une grossesse non désirée.

De même l'utilisation de la CU chez les adolescent(e)s/jeunes ne semble pas poser de problèmes à certains (même s'ils ne sont pas très nombreux), du moment qu'ils s'adonnent à la sexualité.

"Ils sont très actifs sexuellement, donc il faut les sensibiliser et leur permettre de l'utiliser"

(Client, informaticien)

L'utilisation de la CU par les adolescent(e)s devient même une exigence pour garantir leur avenir dans un contexte où l'abstinence est une valeur qui se perd progressivement et à cause de l'influence des valeurs occidentales.

"S'ils ne l'utilisent pas, ils risquent d'avoir des grossesses non désirées ce qui peut gâcher leur vie" (Client, sans emploi)

"On ne peut pas l'empêcher parce que notre société est trop occidentalisée"

(Client, agent de sécurité)

Par contre, la grande majorité des bénéficiaires se prononce contre l'utilisation de la CU chez les adolescent(e) s/jeunes, non seulement parce que cela peut les conduire à la dérive sexuelle, mais aussi parce que cette méthode peut les exposer au IST/VIH/SIDA.

"Cela va développer les IST/SIDA chez les jeunes car ils vont s'adonner à la sexualité à tout bout de champs"

(Client, bijoutier)

\section{ACCESSIBILITÉ DE LA MÉTHODE}

Les propos recueillis auprès des enquêtés révèlent que de nombreuses barrières peuvent limiter ou empêcher l'accessibilité de la CU. Le principal obstacle qui favorise l'inaccessibilité de cette méthode à l'égard des client(e)s est l'ignorance des populations qui est elle-même liée au manque d'informations relatives à ce produit.

"Les gens ne sont pas informés, ils ne connaissent pas la CU"

(Client, élève)

Au-delà de cette méconnaissance due à l'absence de sensibilisation, il y a la gêne, la honte et la peur d'être jugé.

"C'est gênant de se rendre dans une pharmacie pour de pareilles choses"

(Client, élève)

"Les gens ont tendance à traiter de mauvaises personnes celles qui utilisent la CU"

(Apprenti)

Pour quelques enquêtés, même si certaines personnes sont au courant de l'existence de cette méthode, elles peuvent se heurter à d'autres obstacles qui entravent son accès. La qualité de l'accueil et l'attitude négative de certains prestataires des officines n'encourage pas les gens à se rendre dans les pharmacies, comme en témoignent les propos de cet élève (client) : "Les gens ont honte qu'on les dévisage par la suite" 
Outre le manque de confidentialité sur lequel insistent certain(e)s client(e)s "mystère", l'existence de tabous socio culturels et religieux peuvent limiter, surtout chez les jeunes, l'accès à ce produit quand on sait que ce dernier est réservé aux personnes sexuellement actives et que la sexualité hors mariage est fortement réprimandée.

D'autres soulignent l'absence de moyens financiers, surtout chez les jeunes, pour faire face au coût de ce produit qui est jugé un peu cher, ainsi que sa non disponibilité dans certaines structures.

"C'est très cher car n'étant pas à la portée de toutes les filles"

(Client, sans emploi)

"Ce n'est pas disponible dans toutes les pharmacies"

(Cliente, enquêtrice)

Le fait également que la CU ne protège pas contre les IST/SIDA a été identifié comme un facteur pouvant limiter ou empêcher son utilisation.

"La CU ne préserve pas contre les maladies, alors qu'aujourd'hui les sénégalais ont très peur des IST/SIDA" 
Sur les 45 client(e)s "mystère", 26 parmi eux ne comptent pas retourner au niveau des officines privées pour y recevoir une méthode de contraception à cause des insuffisances notées au niveau de l'offre des services et surtout à cause du manque d'informations exhaustives de la part de nombreux prestataires, plus particulièrement par rapport à la CU. De ce fait, ils suggèrent un renforcement des capacités des prestataires des officines à travers des formations.

"Il faut immédiatement renforcer les capacités des vendeurs en pharmacie"

(cliente "mystère")

Ce renforcement des capacités est d'autant plus important que les pharmacies constituent les principales sources d'approvisionnement de cette méthode.

"Il faut aussi des formations au niveau des officines d'autant plus qu'elles pullulent dans les quartiers qui sont les milieux où les gens s'en procurent"

(vendeur en pharmacie)

Pour les bénéficiaires, la promotion du produit doit être assurée au niveau des structures de santé, notamment des centres de PF et des officines privées et s'adresser à la cible adulte pour éviter de heurter certaines sensibilités.

"La CU doit être promue à travers les centres spécialisés, hôpitaux, centres de santé, pharmacies, pour ne pas créer des problèmes avec les religieux et les coutumes sénégalaises"

(Commerçante)

Ils suggèrent également de la rendre disponible dans les pharmacies et que son coût soit revu à la baisse. 


\section{ECOMMANDATIONS}

Pour améliorer l'offre de services de CU dans les officines privées, de nombreuses solutions ont été suggérées et sur la base desquelles 5 recommandations essentielles ont été formuler à savoir :

1. Orienter/former les prestataires des pharmacies privées sur les nouveaux protocoles en matière d'offre de CU;

2. Renforcer la sensibilisation sur la CU;

3. Améliorer l'accessibilité financière de la CU;

4. Assurer la promotion de la CU au niveau des pharmacies (affiches, dépliants...) ;

5. Mettre à la disposition des prestataires des pharmacies privées les nouveaux protocoles. 


\section{ANNEXE 1 : TABLEAU SORTIES DE NORLEVO ${ }^{\circledR}$ POUR LE 1ER SEMESTRE 2009 (SODIPHARM)}

\begin{tabular}{|l|c|}
\hline Mois & Norlevo $^{{ }^{\circ}}$ \\
\hline Janvier & 256 \\
\hline Février & 1149 \\
\hline Mars & 1156 \\
\hline Avril & 744 \\
\hline Mai & 941 \\
\hline Juin & 1211 \\
\hline Juillet & 1300 \\
\hline
\end{tabular}

NB : Stock disponible an 31 juillet 2009, 974 boittes.

\section{ANNEXE 2 : DÉCLARATION DE POLITIQUE (JUILLET 2003) DU CONSORTIUM INTERNATIONAL POUR LA CU Mise à jour sur le régime}

\section{Dosage et posologie}

De récentes études ont apporté de nouvelles informations sur le régime des pilules contraceptives d'urgence (PCU) au lévonorgestrel seul. D’après les résultats, une dose unique de $1,5 \mathrm{mg}$ de lévonorgestrel peut remplacer deux doses de $0,75 \mathrm{mg}$ prises à 12 heures d'intervalle. Selon de nouvelles recherches, il semblerait que les PCU (aussi bien la formule au lévonorgestrel que la méthode de Yuzpe) permettent d'éviter une grossesse jusqu'à 5 jours (120 heures) après un rapport sexuel non protégé.

- Une dose unique de 1,5 mg (PCU au lévonorgestrel seul)

D’après une étude multi centre randomisée réalisée par l’Organisation Mondiale de la Santé (OMS) dans dix pays du monde développé et en développement, la dose unique de 1,5 $\mathrm{mg}$ de lévonorgestrel est aussi efficace pour prévenir une grossesse que deux doses de $0,75 \mathrm{mg}$ prises à 12 heures d'intervalle. Les effets secondaires ne diffèrent pas d'une formule à l'autre.(1) Une étude nigériane a corroboré le résultat selon lequel une dose unique de 1,5 mg de lévonorgestrel est à la fois efficace et sans danger.(2) La formule à dose unique simplifie l'utilisation du lévonorgestrel pour la contraception d'urgence.

- La prise de PCU devrait intervenir dès que possible mais peut se faire jusqu'à 5 jours (120 heures) après un rapport sexuel non protégé

La contraception d'urgence au lévonorgestrel seul est efficace pour prévenir une proportion élevée de grossesses jusqu’à 5 jours (120 heures) après un rapport sexuel non protégé, selon les résultats d'une étude randomisée multi centre réalisée par l'OMS.(1) La formule oestrogène et progestatif combinés (méthode de Yuzpe) réduit aussi le risque de grossesse jusqu'à 5 jours, selon les résultats d'une étude canadienne.(3)

Cependant, les résultats de l'étude de l'OMS révèlent une tendance significative à la baisse de l'efficacité à mesure que s'agrandit le délai entre le rapport sexuel non protégé et la prise de la PCU, et des études précédentes de l'OMS ont indiqué une augmentation du risque de grossesse à mesure que la prise de PCU est retardée.(1-4) Ces résultats soulignent à quel point il est important de fournir une PCU aux femmes qui en expriment le besoin au-delà de 72 heures. Pour optimiser l'efficacité de la méthode, il conviendrait néanmoins d'encourager les femmes à prendre la PCU le plus tôt possible après un rapport sexuel non protégé. Les PCU sont inefficaces après implantation de l'ovule fécondé.

\section{Recommandation}

Bien que la présentation du produit et les notices d'information fournies par les fabricants des PCU soient peu susceptibles de changer dans un avenir immédiat, les fabricants sont encouragés de mettre à jour leurs protocoles PCU afin qu'ils reflètent ces nouvelles informations. Sur la base des données les plus récentes, les prestataires devraient recommander aux femmes la formule de PCU à dose unique de 1,5 $\mathrm{mg}$ de lévonorgestrel seul. Ils devraient continuer de promouvoir la prise de PCU le plus tôt possible suivant un rapport sexuel non protégé mais offrir les PCU dans un délai maximum de 120 heures après un rapport sexuel non protégé, selon les besoins. 


\section{ANNEXE 3 : AUTORISATION D'ENQUÊTE}

Mr le Directeur de la Pharmacie

Dans le cadre d'une étude sur la contraception d'urgence, une enquête sera menée au niveau de 15 officines privées sélectionnées dans la région de Dakar.

L'objectif de l'enquête est de mettre en place une stratégie qui permet d'améliorer l'offre de la contraception d'urgence aux clients(es) à travers un counseling et une orientation vers d'autres services de santé de la reproduction pour une prise en charge appropriée.

Pour ce faire, les enquêteurs se rendront auprès des prestataires des officines du 13 au 17 Juillet 2009 afin de collecter les informations relatives à l'étude.

La disponibilité des prestataires ainsi que des données sur la contraception d'urgence contribueront à l'atteinte des objectifs de l'enquête.

Nous comptons sur votre appui, afin de démarrer cette étape de l'étude sur la contraception d'urgence.

Dans l'attente d'une suite favorable, nous vous remercions de votre franche collaboration et vous prions de croire, $\mathrm{Mr}$ le Directeur à l'expression de nos sincères remerciements. 


\section{IBLIOGRAPHIE}

\section{Population Council/CEFOREP}

"Etude sur les possibilités d'introduction de la contraception d'urgence au Sénégal".

Mai 2003.

Population Council Nairobi/ECafrique

"Study on bridging users of Emergency Contraceptives to other Family Planning and Reproductive Health services through private sector pharmacies" .

July 2008 - February 2009.

Enquête Démographique et de Santé 2005 (EDS)

Rapport préliminaire. Ministère de la Santé et de la Prévention Médicale.

Centre de Recherche pour le Développement Humain. Dakar, Sénégal.

\section{OMS}

"guide pour la conduite d'évaluation visant à élargir le choix des méthodes contraceptives et à améliorer la qualité des soins".

Genève 2002.

DSR/Population Council

"Etude Diagnostique sur la Contraception d'Urgence".

Février 2006 


\section{ITE WEB}

\section{SITE WEB}

Consortium International pour la Contraception d'Urgence

ECafrique : mmw.ecafrique.org

Population Council : wmm.popcouncil.org

CEFOREP : www.ceforep.orange.sn 
